\title{
Calcium Phosphate Cement in the Surgical Management of Benign Bone Tumors
}

\author{
TAKASHI HIGUCHI, NORIO YAMAMOTO, KATSUHIRO HAYASHI, \\ AKIHIKO TAKEUCHI, HIROAKI KIMURA, SHINJI MIWA, KENTARO IGARASHI, \\ KENSAKU ABE, YUTA TANIGUCHI, HISAKI AIBA and HIROYUKI TSUCHIYA
}

Department of Orthopaedic Surgery, Graduate School of Medical Science, Kanazawa University, Kanazawa, Japan

\begin{abstract}
Background/Aim: This study assessed the mid- to long-term outcomes of calcium phosphate cement (CPC) implantation in benign bone tumor surgery. Patients and Methods: Between 2000 and 2015, 130 patients underwent CPC implantation in benign bone tumor surgery. Radiographic findings and clinical outcomes were retrospectively evaluated. Results: The mean follow-up period was 52 months. CPC filling immediately after surgery was sufficient, regardless of the amount of CPC used and the usage of adjuvant substances, which resulted in $92 \%$ of the patients' radiological results being classified as good or excellent. Significantly more patients had better CPC filling among patients with less hemorrhage and patients with tourniquet. The number of patients with good or excellent CPC filling had significantly increased by the final followup. Conclusion: CPC is a useful bone substitute for benign bone tumor surgery providing excellent osteoconductivity and long-lasting stability without internal fixation.
\end{abstract}

Autologous bone is frequently implanted after curettage of benign bone tumors, and is the gold standard of filling as augmentation, guaranteeing rapid graft incorporation and bone remodeling $(1,2)$; however, there is a limit to the amount that can be used and it is unsuitable for repairing large defects. Allogenous bone and bone cement [polymethyl methacrylate (PMMA)] are used to replace large defects, and internal fixation methods, such as plates, screws, or intramedullary nail fixation, are often used in combination to increase stability $(1,3,4)$. To make surgery less invasive, deficient areas can be filled with calcium phosphate bone

Correspondence to: Akihiko Takeuchi, Department of Orthopaedic Surgery, Graduate School of Medical Science, Kanazawa University, 13-1 Takara-machi, Kanazawa 920-8641, Japan. Tel: +81 762652374, Fax: +81 762344261, e-mail: a_take@med.kanazawa-u.ac.jp

Key Words: Calcium phosphate cement, benign bone tumor, radiological outcomes, complication. paste (CPC) after benign bone tumor curettage. CPC acts as a synthetic osteoconductive scaffold and reaches its peak strength within a few days after implantation in bone, meaning that it can be used to fill large defects without the need for additional fixation and making it an attractive alternative to autologous bone implantation (5-7). While the use of CPC in orthopedic surgery is well described in traumatology in several reports with long follow-up periods (8-10), few data are available on the use of CPC in bone tumor surgery $(2,11)$. The present retrospective study evaluated mid- to long-term outcomes of CPC implantation in patients undergoing benign bone tumor surgery.

\section{Materials and Methods}

Between 2000 and 2015, 130 patients (79 men, 61 women; mean age $=31.6$ years; range $=6-77$ years) underwent $\mathrm{CPC}$ implantation into bone defects after curettage of benign bone tumors (101 primary cases, 29 recurrent cases). Histological types included bone giant cell tumors (GCTs) in 44, simple bone cysts (SBCs) in 28 , enchondroma in 16, chondroblastoma in nine, fibrous dysplasia in eight, non-ossifying fibroma in four, intraosseous ganglion in four, aneurysmal bone cysts (ABCs) in three, and other types in 14. In cases of a locally aggressive bone tumor including GCTs and ABCs, adjuvant treatments using shaving with a high-speed burr and phenol and ethanol treatment were provided. In cases of SBC, multiple holes were drilled into the cancellous bone. These adjuvant treatments were performed in 78 patients. One patient who presented with a pathological fracture and another who was at high risk of fracture were treated with screw fixation in addition to CPC grafting. Surgical sites included the upper limb bones in 37 , the pelvic bones in 17 , and the lower limb bones in 76 . Operations were performed with a tourniquet in 79 patients and without a tourniquet in 51 patients. For CPC, BIOPEX or BIOPEX-R (HOYA Technosurgical Corp., Tokyo, Japan) was used in 113 patients and CERAPASTE (NGK SPARK PLUG Co., Ltd., Nagoya, Japan) was used in 17 patients.

Radiographic findings obtained immediately after surgery and at final follow-up were classified into four radiographic categories for adequacy of CPC filling according to Matsumine classification (5): poor: clear zone completely surrounding the CPC; acceptable: clear zone occupying $>50 \%$ of the CPC margin; good: clear zone 


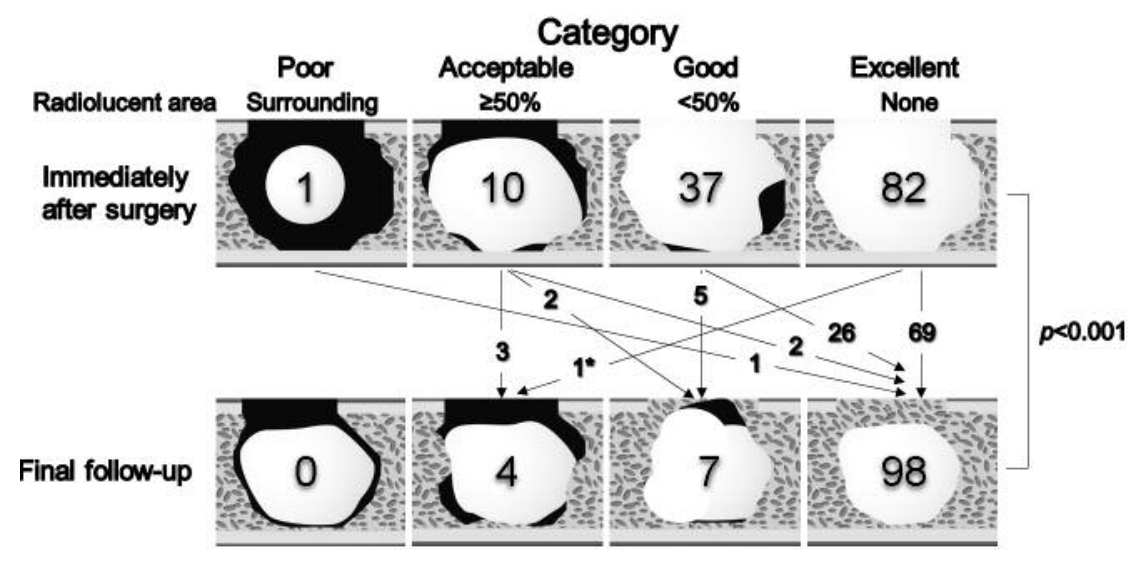

Figure 1. Adequacy of filling of defects with calcium phosphate bone paste (CPC) after surgery for benign bone tumor immediately after surgery and at final follow-up. All patients were categorized into four radiographic categories according to the proportion of radiolucent area after CPC filling of defects. Patients who experienced recurrence were excluded. The number of patients with better CPC filling had significantly increased by the final follow-up (Wilcoxon signed-rank test; $p<0.001$ ). Numbers indicate the number of patients. Asterisk indicates a patient with postoperative fracture.

occupying $\leq 50 \%$ of the CPC margin; or excellent: no clear zone around the CPC (Figure 1).

Radiographic CPC filling immediately after surgery and at final follow-up were compared using the Wilcoxon signed-rank test. To evaluate factors affecting adequacy of CPC filling, radiographic CPC filling immediately after surgery was compared according to amount of CPC used ( $\geq 30 \mathrm{~g}$ or $<30 \mathrm{~g}$ ), intraoperative hemorrhage $(\geq 100 \mathrm{ml}$ or $<100 \mathrm{ml}$ ), tourniquet use, and adjuvant therapy utilization using the Mann-Whitney $U$-test. Clinical findings including postoperative complications, pain, limb function, and time to full weight bearing were evaluated. The statistical analyses were performed with EZR (Saitama Medical Center, Jichi Medical University, Saitama, Japan) (12). This retrospective clinical case series study was approved by the local Institutional Review Board. All patients were informed that the data from this study would be submitted for publication and they provided their consent for participation.

\section{Results}

The mean follow-up period was 32 months (range $=2-145$ months). The mean amount of CPC used was $29 \mathrm{ml}$ (range $=1-129 \mathrm{ml}$ ) and the mean surgical time was 115 minutes (range $=35-365$ minutes). Radiographic CPC filling immediately after surgery was classified as poor in one, acceptable in 10 , good in 37 , and excellent in 82 cases (Figure 1). CPC filling was significantly worse in those with pronounced hemorrhage $(\geq 100 \mathrm{ml})(p=0.02)$, although there was no significant difference in CPC filling between groups which used a large CPC amount $(\geq 30 \mathrm{~g})$ and those using a small CPC amount $(<30 \mathrm{~g})(p=0.06)$. Moreover, CPC filling was significantly better when a tourniquet was used $(p=0.04)$ and was not influenced by adjuvant therapy use $(p=0.14)$ (Figure 2). Radiographic CPC filling at the final follow-up (excluding cases with postoperative recurrence) was classified as poor in 0 , acceptable in four, good in seven, and excellent in 98 cases, and was significantly improved compared to evaluation immediately after surgery $(p<0.001)$ (Figure 1$)$.

Among the four patients with only acceptable results on radiographs at the final follow-up, one (from excellent to acceptable) experienced a partial bone fracture after the surgery, whereas the other three were cases with short follow-up, with a mean follow-up period of 2.7 months.

Complications such as noninfectious discharge lasting longer than a week in 10 , hydrarthrosis in eight, postoperative fever $>38.5^{\circ} \mathrm{C}$ persisting for more than 4 days in five, superficial wound infection in one, and synovitis in one case were observed; all cases healed through conservative treatment. Postoperative bone fracture was encountered in three patients. Among them, one patient with a distal phalanx enchondroma and a thin bone cortex was subsequently treated with pinning; one patient with recurrent GCT of the proximal femur suffered a partial fracture and was treated with CPC re-filling; and one patient with recurrent GCT of the distal radius who suffered a partial fracture and presented with Kellgren-Lawrence grade 4 osteoarthritis after the third operation for recurrence was treated conservatively. Twentyone patients (nine with GCTs, seven with SBCs, two with ABCs, two with fibrous dysplasia, and one with chondroblastoma) displayed local recurrence, although 11 (six with SBCs, five with GCTs) were initially recurrent cases. None of the patients reported pain at the surgical site, limited range of motion in the adjacent joints, or bone deformity. Regarding the 16 patients in whom the remnant of the CPC in the soft tissue was detected radiographically immediately after surgery, it had disappeared in 13 at the final follow-up and the other three were asymptomatic. The mean period to full weight bearing was 17 days. 


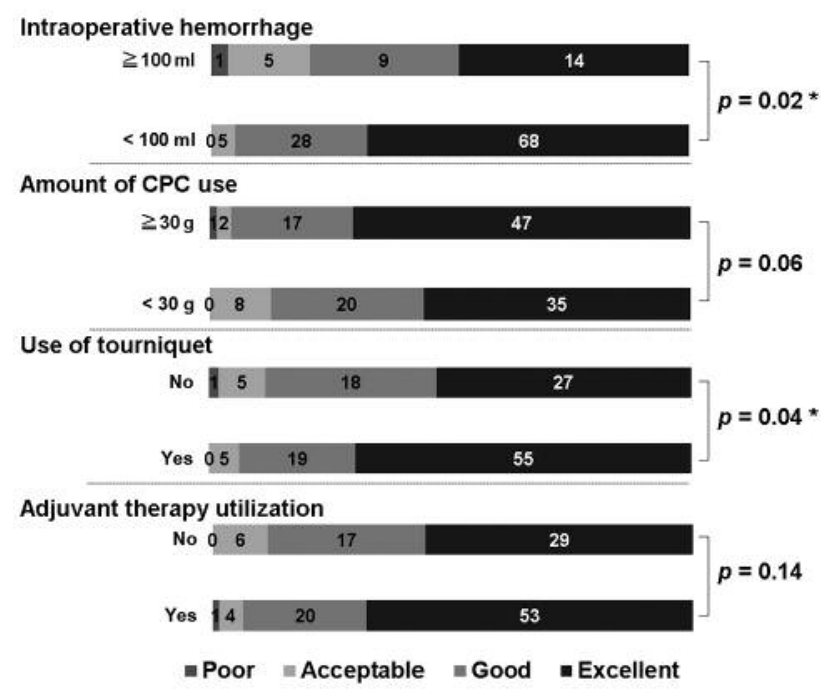

Figure 2. Adequacy of filling of defects with calcium phosphate bone paste (CPC) after surgery for benign bone tumor according to different factors. Radiographic categories were compared immediately after surgery according to intraoperative hemorrhage, amount of CPC use, use of tourniquet and utilization of adjuvant therapy. CPC filling was better in patients with less hemorrhage and with tourniquet use (*Mann-Whitney $U$-test; $p<0.05)$. There was no significant difference in the number of patients with better CPC filling according to amount of CPC used and the use of adjuvant therapy categories. Numbers in the bars indicate the number of patients.

\section{Discussion}

Extended curettage is the most common treatment for benign bone tumors and lytic lesions (1-5); as a result, large bone defects with a risk of fracture sometimes occur in cases with large tumors. PMMA cement is frequently used to fill large defects $(3,4)$, however, it only fills the space, as it has no bioactive or intrinsic adhesive properties. Therefore, PMMA provides only instant and temporal stability and should be used in conjunction with an internal fixture to prevent postoperative fracture (3). In addition, the polymerization of PMMA is an exothermic process that can induce tissue necrosis and can lead to the appearance of a radiolucent area around the cement that can reduce stability and increase the risk of postoperative fracture of the cemented site (Figure 3) $(3,5)$. The exothermic process can also damage the articular cartilage and induce future osteoarthritic changes in the adjacent joint, especially in cases in which less subchondral bone remains $(3,13)$. Benevenia et al. reported on 22 patients with GCTs treated with curettage and PMMA insertion, 55\% of whom suffered non-oncological complications, including five cases of periarticular fractures and seven cases of osteoarthritis progression, despite $64 \%$ having been treated with additional pin or plate fixation (3). In the present study, there was no case of osteoarthritis progression during follow-up except for one case with recurrent GCT of the distal radius treated with three operations for recurrence and partial bone fracture because of CPC setting in situ at physiological temperatures $(5,6)$. Apart from this patient, only two more patients, with aggressive GCT and enchondroma, experienced postoperative fractures in the present study despite the low number of cases with additional fixation or the existence of large CPC-filled cavities.

The advantages of CPC include its high osteoconductivity in addition to its fast self-setting time and high moldability $(6,7)$. Marukawa et al. reported that new bone formation and bone morphogenic protein-2-stained cells were detected around the implanted screw which was inserted into the nasal bone of rabbits in combination with CPC from 4 weeks after implantation and were significantly increased compared to the group treated without CPC (6). In the present study, the CPC filling was insufficient in 11 out of 130 cases immediately after surgery, cases that were classified as poor or acceptable radiographically. However, in all cases but four with short follow-up, the radiolucent areas and even the cortical window were replaced with new bone by the final follow-up (Figures 1 and 4), which demonstrates the highly osteoconductive activity of CPC.

The possibility of in situ physical breakdown prior to CPC fully setting, the so-called washout phenomenon, must be mentioned as a disadvantage of CPC $(5,14)$. Blood contamination of $\mathrm{CPC}$ can weaken the setting reaction, and such blood-contaminated CPC can flow out to soft tissue and be the cause of insufficient CPC filling or of complications associated with soft-tissue inflammation $(5,14)$. In the present study, CPC filling immediately after surgery was significantly worse in those with extensive intraoperative hemorrhage. Furthermore, there were 25 cases of minor complications associated with the washout phenomenon; however, the incidence of complications was low and all were minor that healed conservatively. In order to reduce the washout phenomenon, the following procedures are recommended: i) hemostasis should be performed carefully, using a tourniquet if possible, as in the present series, CPC filling immediately after surgery was better in the group with tourniquet use; ii) CPC with a firm clay-like stiffness should be created by reducing the liquid component from that usually recommended; iii) the CPC should be strongly compressed against the cavity wall using the operator's thumb to prevent hemorrhaging from the curetted bone; iv) the soft tissue should be washed with sufficient physiological saline to remove blood-contaminated CPC after sealing the bony cavity with cortical bone. While blood contamination can reduce the strength of the hardened CPC, it is not expected to ultimately become a problem because it can stimulate CPC absorption and bone replacement (7). An improvement in absorbency and bone replacement due to blood addition were confirmed in the in vivo study of Musha et al., which reported that the blood penetrates the surface of 


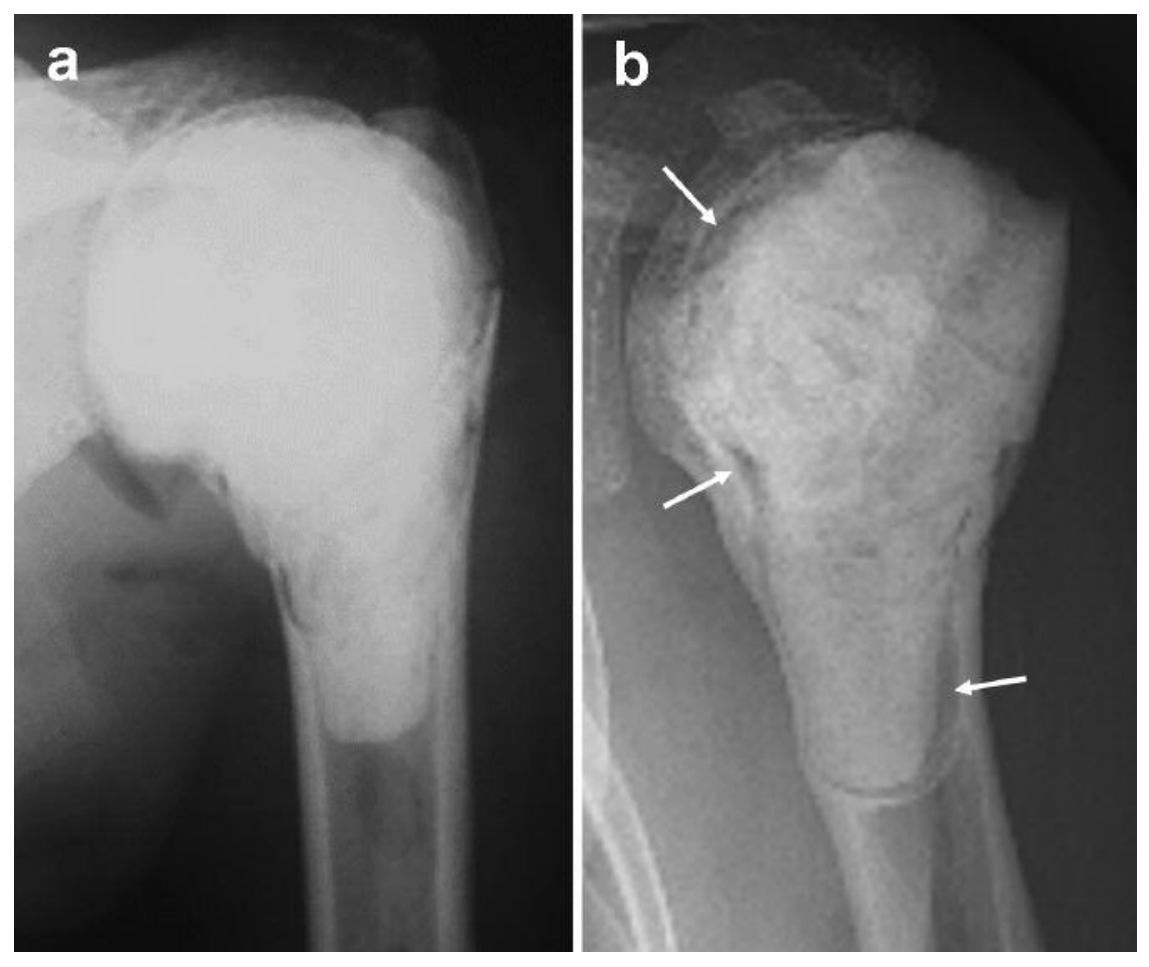

Figure 3. A 24-year-old woman with a giant cell tumor in the left proximal humerus. a: Radiograph immediately after surgery. Polymethyl methacrylate (PMMA) cement was packed into the cavity after curettage of the tumor. b: Radiograph 4 years after surgery showing a radiolucent area around the PMMA cement (white arrows).

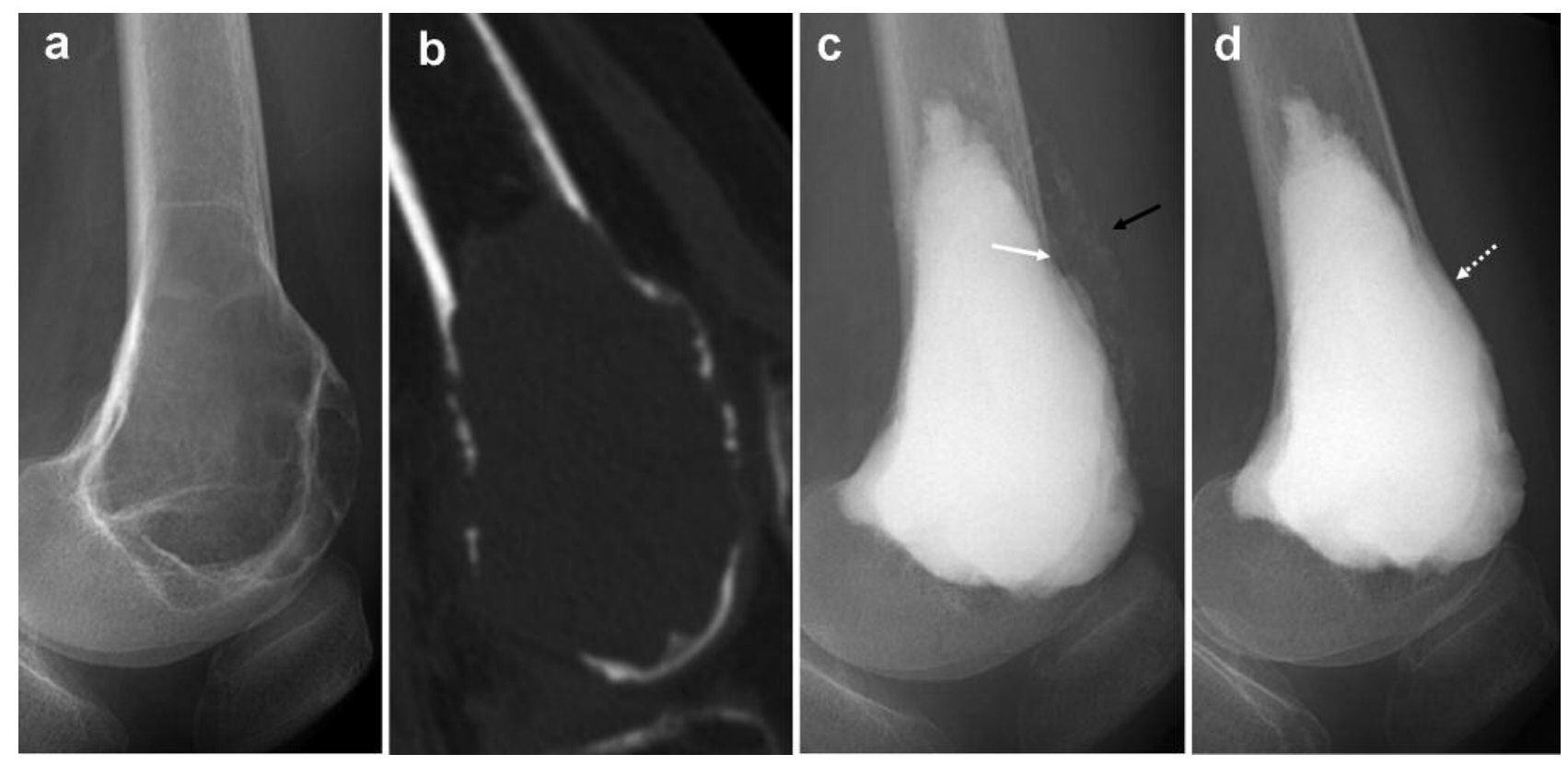

Figure 4. $a, b$ : A 59-year-old woman with a giant cell tumor in the left distal femur. c: Radiograph immediately after surgery. In total, the cavity was filled with $59 \mathrm{~g}$ of calcium phosphate cement $(C P C)\left(B I O P E X^{\circledR}\right)$ after curettage of the tumor. A cortical defect (white arrow) and remnant of $C P C$ at the soft tissue (black arrow) were detected. $d$ : Radiograph 5 years later showing that the remnant of CPC had disappeared and the cortical defect had completely healed (dotted arrow). 
CPC and stimulates hardened CPC to become more porous, leading to early CPC absorption and bone replacement (7). In the present study, most of the areas with insufficient CPC filling group with extensive hemorrhage had been replaced with new bone by the final follow-up. In addition, most of the CPC remnants in the soft tissues were confirmed radiographically immediately after the surgery to have been absorbed and had disappeared by the final follow-up (Figure 4). However, surgeons must carefully manage weight bearing in the early postoperative period.

In conclusion, although a few complications were encountered, CPC appears to be a useful bone substitute for the treatment of benign bone tumors. Osteoconductivity of CPC provides long-lasting stability without the need for additional fixation, enabling for lower invasiveness. Blood contamination should be monitored because it can weaken CPC setting, resulting in insufficient CPC filling and complications related to soft-tissue inflammation; however, neither will cause problems during follow-up because of the high osteoconductivity of CPC and increased absorption in the case of blood contamination of CPC.

\section{References}

1 Parrish FF: Treatment of bone tumors by total excision and replacement with massive autologous and homologous grafts. J Bone Joint Surg Am 48: 968-990, 1966.

2 Nakamura T, Matsumine A, Asanuma K, Matsubara T and Sudo A: Treatment of bone defect with calcium phosphate cement subsequent to tumor curettage in pediatric patients. Oncol Lett 11: 247-252, 2016.

3 Benevenia J, Rivero SM, Moore J, Ippolito JA, Siegerman DA, Beebe KS and Patterson FR: Supplemental bone grafting in giant cell tumor of the extremity reduces nononcologic complications. Clin Orthop Relat Res 475: 776-783, 2017.

4 Wallace MT, Henshaw RM: Results of cement versus bone graft reconstruction after intralesional curettage of bone tumors in the skeletally immature patient. J Pediatr Orthop 34: 92-100, 2014.

5 Matsumine A, Kusuzaki K, Matsubara T, Okamura A, Okuyama N, Miyazaki S, Shintani K and Uchida A: Calcium phosphate cement in musculoskeletal tumor surgery. J Surg Oncol 93: 212220, 2006.
6 Marukawa K, Ueki K, Okabe K, Ueki K, Okabe K, Nakagawa $\mathrm{K}$ and Yamamoto $\mathrm{E}$ : Use of self-setting $\alpha$-tricalcium phosphate for maxillary sinus augmentation in rabbit. Clin Oral Implants Res 22: 606-612, 2011.

7 Musha Y, Umeda T, Yoshizawa S, Shigemitsu T, Mizutani K and Itatani K: Effects of blood on bone cement made of calcium phosphate: problems and advantages. J Biomed Mater Res B Appl Biomater 92: 95-101, 2010.

8 Russell TA, Leighton RK and Alpha-BSM Tibial Plateau Fracture Study Group: Comparison of autogenous bone graft and endothermic calcium phosphate cement for defect augmentation in tibial plateau fractures. A multicenter, prospective, randomized study. J Bone Joint Surg Am 90: 2057-2061, 2008.

9 Bohner M: Calcium orthophosphates in medicine: from ceramics to calcium phosphate cements Injury 31: 37-47, 2000.

10 Verlaan JJ, van Helden WH, Oner FC, Verbout AJ and Dhert WJ: Balloon vertebroplasty with calcium phosphate cement augmentation for direct restoration of traumatic thoracolumbar vertebral fractures. Spine 27: 543-548, 2002.

11 Takeuchi A, Suwanpramote P, Yamamoto N, Shirai T, Hayashi K, Kimura H, Miwa S, Higuchi T, Abe K and Tsuchiya H: Midto long-term clinical outcome of giant cell tumor of bone treated with calcium phosphate cement following thorough curettage and phenolization. J Surg Oncol, 2018. doi: 10.1002/jso.24971 [Epub ahead of print]

12 Kanda Y: Investigation of the freely-available easy-to-use software "EZR" (Easy R) for medical statistics. Bone Marrow Transplant 48: 452-458, 2013.

13 Suzuki Y, Nishida Y, Yamada Y, Tsukushi S, Sugiura H, Nakashima $\mathrm{H}$ and Ishiguro $\mathrm{N}$ : Re-operation results in osteoarthritic change of knee joints in patients with giant cell tumor of bone. Knee 14: 369-374, 2007.

14 Lu J, Descamps M, Dejou J, Koubi G, Hardouin P, Lemaitre J and Proust JP: The biodegradation mechanism of calcium phosphate biomaterials in bone. J Biomed Mater Res 63: 408412, 2002.
Received March 15, 2018

Revised April 9, 2018

Accepted April 12, 2018 